\title{
OpenAIRE FP7 Post-Grant Pilot: A Summary of the Main Results
}

\author{
Gwen Franck
}

\section{Introduction}

1 The OpenAIRE FP7 Post Grant Open Access Pilot, originally intended to end on 30 April 2017, had been granted a 10 month extension and has come to an end in February 2018. In the following, you will read a report on the uptake and metrics of this extension period together with the overall statistical analysis of the Pilot activities. As the Pilot is now officially "closed", in an effort to compile as complete a dataset as possible, some "old" data previously not analysed has been included here. Even now, there are still a couple of publications "pending" because the accounting process has not been finalised. When author fee data (this can be an estimate) is already available, the data has already been added to the bulk dataset and the following analysis but they might not be visible in the statistical module of the Pilot website. As none of these pending data significantly differs from the data provided in the system, we do not believe that this will influence the statistical analysis below, although individual numbers (such as amount of articles funded per publisher) might change a little bit. As the final data will be processed, we will provide a final version of the dataset as an addition to the existing datasets available on Zenodo. $^{1}$

\section{The Pilot}

2 The Pilot was installed by OpenAIRE and the European Commission in order to meet demand from FP7 projects that wanted, in compliance with the EC's recommendations and polices on open access, to make their project outputs available as open access, but did not have the funding appropriated in their project budgets. Up to three publications per project were eligible for funding, provided the acceptance date of the publication dates after the project closing date. A total budget of $€ 4,000,000,000$ has been made available 
fort his, of which approximately $€ 2,650,000$ has been spent (including $€ 400,000$ for a supporting mechanism for non-author fee based publishing initiatives, not further included in this report). ${ }^{2}$

In total, the Pilot has funded 1,323 publications, 1,232 articles, 71 monographs, 18 book chapters and 2 conference proceedings. Out of these, 186 have been funded via prepayment agreements with publishers and 25 have been processed through block grants with two university libraries.

4 The Pilot had installed a funding cap of $€ 6,000$ for monographs and $€ 2,000$ for all other publication types (mainly articles). If the original author fee exceeded this amount, the submitter was asked to provide a partial invoice or request for reimbursement that did not exceed this amount.

5 The average author fee for articles processed is $€ 1,474$ and the median fee is $€ 1,446$. Both amounts are well below our funding cap. The Pilot only funded articles that appeared in "full" open access journals. Hybrid journals, that offer a mix of subscription based and open access articles, have been explicitly excluded from funding, which explains the relatively lower share of some well-known legacy publishers offering mainly hybrid journals in these statistics.

6 The Pilot has installed pre-payment agreements with 5 publishers (Wiley, Copernicus, BioMedCentral, BMJ and Ubiquity Press). A total of approx. $€ 317,000$ has been spent on 186 publications. In close collaboration with the Pilot administration, these publishers identified eligible publications and added them to their account-applying a discount on the listed author fee. Additionally, 2 university libraries (Radboud University in the Netherlands and Bielefeld University in Germany) each have received a block grant from OpenAIRE-allowing them to identify and fund eligible publications without going through the invoicing process in the Pilot system. In total, 25 publications have been supported this way. A separate dataset compiling these numbers will be added to the Zenodo record. 


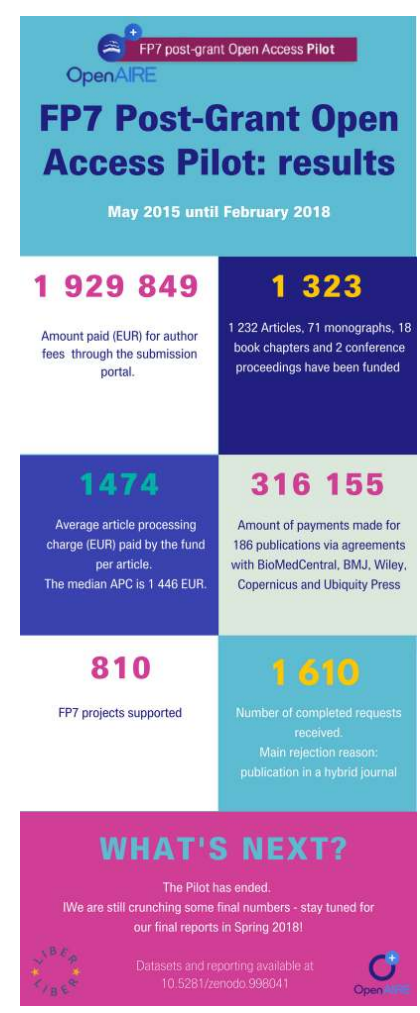

\section{Conclusions}

7 This Pilot has proven that there is definitely a demand for funder-driven support for author fee payments. With over 800 FP7 projects making use of this Fund for in total more than 1300 publications over a period of 34 months, the Pilot has, after a slow start, gained a certain traction. Thanks to targeted dissemination actions by the Pilot administration, open access publishers, library and repository managers and the OpenAIRE NOADs, this funding scheme has become widely known as a reliable source of funding for the time it was in place.

Here lies the first "weakness" of the Fund: as it became more widely known, the fact that the Pilot was limited in time and scope (FP7 projects, post-grant) led to some frustration by ineligible projects. The expectation that this Pilot would transform into a structural support mechanism has been expressed by both beneficiaries and rejected applicants. The Pilot still receives requests for funding (via e-mail) by people unaware that this support is not a permanent service.

Perhaps surprisingly, the rule that the Pilot would not support hybrid journals was met with relatively little resistance. The Pilot administration has always taken great care explaining why it did not support this type of journals (as it did not want to support "double dipping" using public funds) and usually applicants understood. Either because they did not know their journal of choice was hybrid, or because with certain funders this hybrid status is not an impediment to funding, this could lead to some sour reactions. However, we believe that the "no-hybrid" approach has proven its benefits, and it has definitely raised awareness among researchers about this issue. If anything, it shows the need for more education on the different forms of open access publishing, as in some 
cases journals and publishers remain deliberately unclear about their hybrid status (using terms like "author choice").

As the Pilot has experimented with pre-payment agreements for certain publishers and block grants for libraries, some lessons can be learnt from this experience as well. From the start, the Pilot has imposed firm conditions on the publishers involved in funding eligibility, including the application of a bulk discount for all publications processed via this system. However, the Pilot has been very accommodating when reporting was concerned, allowing different channels of evaluation (such as dashboards or spreadsheets) and timing of data delivery (sometimes at the moment of submissions, sometimes only when a publication was in its last editorial stage before publishing). The experience from this Pilot learns that, when entering into agreements with publishers, a suitable and uniform reporting mechanism has to be provided by the funder-ideally processing the data in a similar fashion and at the same time as individual submissions.

11 Another crucial element that proved to be rather difficult to execute well, was the rather complicated invoicing procedure. No matter how many FAQs, information sheets and webinars delivered on the subject, the number of submissions that got it "right" from the first time were a minority. Invoices contained VAT, or were addressed to the researcher rather than to the OpenAIRE admin, or exceeded our funding caps. The bulk of the administrative effort consisted of fixing these errors, often leading to tiresome back-andforths between researchers, their administration, Pilot administration, the Pilot accounting office and the publishers issuing the invoices.

Finally, two remarks about the critique that this Pilot encourages the author fee based route in open access publishing. The Pilot has funded 17 non-author fee open access initiatives in order for them to be able to solidify their activities both on a technical and a sustainability level.

Despite stressing that this Fund is indeed a "pilot", of which all experiences-also the negative ones-should be taken into account when undertaking similar efforts in the future, some have reported that the mere existence of this Fund has lead to an increase in author fee based publishing initiatives. One can only hope that the firm rules set by this Pilot (the funding caps and the "no hybrid" rules), have at least shown that it is possible to go about author fee based publishing in a non-predatory way. Many publishers have not only shown a lot of eagerness and willingness to collaborate with the conditions set by the Pilot, but as a detailed analysis of the dataset shows, also show a lot of flexibility when it comes to the actual author fees being charged. This lead to the conclusion that there is a lot of room for funders to set the rules when it comes to reimbursing author fees, rather than simply following the major players in the market.

\section{NOTES}

1. DOI: http://doi.org/10.5281/zenodo.1219084. We explicitly invite interested parties to conduct their own analysis based on the original dataset. It is also possible to download a csv with all data directly from the Pilot website https://postgrantoapilot.openaire.eu. 
2. An initial report of the funded initiatives is available on the OpenAIRE blog https:// blogs.openaire.eu/?p=2754

\section{ABSTRACTS}

This short article aims at presenting the main outcomes of the OpenAIRE FP7 post-grant pilot, an initiative from the European Commission to cover publication costs after the end of the projects.

INDEX

Keywords: OpenAIRE, FP7 post-grant Open Access pilot, Article Processing Charges

\section{AUTHOR}

\section{GWEN FRANCK}

LIBER, Netherlands

gwen.franck@kb.nl 